Saudi Journal of Medical and Pharmaceutical Sciences

Abbreviated Key Title: Saudi J Med Pharm Sci ISSN 2413-4929 (Print) |ISSN 2413-4910 (Online) Scholars Middle East Publishers, Dubai, United Arab Emirates Journal homepage: https://saudijournals.com/sjmps

\title{
Presumed Central Serous Chorioretinopathy in 9 Years Old Boy
}

Omer Othman Abdullah

M.B.Ch.B/MD/FICO VR, Vitreoretinal Surgeon and Medical Retina Specialist, Phacoemulsification trainer, Ibinsina Modern Eye and Retina Center, Ministry of Health, Erbil, Iraq

DOI: $10.36348 /$ sjmps.2020.v06i06.006

| Received: 07.06.2020 | Accepted: 16.06.2020 | Published: 24.06.2020

*Corresponding author: Omer Othman Abdullah

\section{Abstract}

A 9-year-old male attended our clinic with the blurred vision in his right eye, for one day duration. No past history of systemic or ocular illnesses except for recurrent tonsillitis. On ocular examination; his left eye (OS) visual acuity (VA) was $6 / 6$, and the right eye (OD) VA was $6 / 9.5$ which corrected to $6 / 9$ with +0.75 diopter sphere. Fundus examination revealed normal optic nerve and a serous detachment in the posterior pole, nearly about the disc size. The optical coherence tomography angiography (OCTA) showed neurosensory retinal detachment with subretinal fluid. The family was not able to perform the fundus fluorescein angiography (FFA) due to the financial crisis in the region. Given this background, the diagnosis of idiopathic central chorioretinopathy had given. The clinical course was monitored without any treatments and spontaneous remission achieved within six months Figure 1, 2 and 3.

Keywords: Central serous chorioretinopathy, children, spontaneous remission of Central serous chorioretinopathy.

Copyright @ 2020: This is an open-access article distributed under the terms of the Creative Commons Attribution license which permits unrestricted use, distribution, and reproduction in any medium for non-commercial use (NonCommercial, or CC-BY-NC) provided the original author and source are credited.

\section{CASE RePORT}

The secondary causes had been excluded by pediatrician and endocrinologist as follows:

1. Complete blood picture plus erythrocyte sedimentation rate (ESR) was normal.
2. Serum cortisol was normal $(18 \mathrm{mcg} / \mathrm{L})$, at $8: 30$ AM.

3. Mean arterial blood pressure was $110 / 65 \mathrm{~mm}$ $\mathrm{Hg}$.

4. No abnormality in the central nervous system.

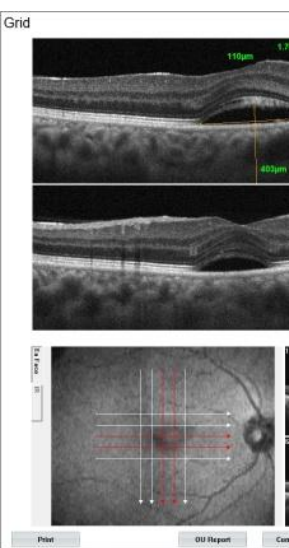

Fig-1: OCTA of the right eye. Showing Central Serous Choriore

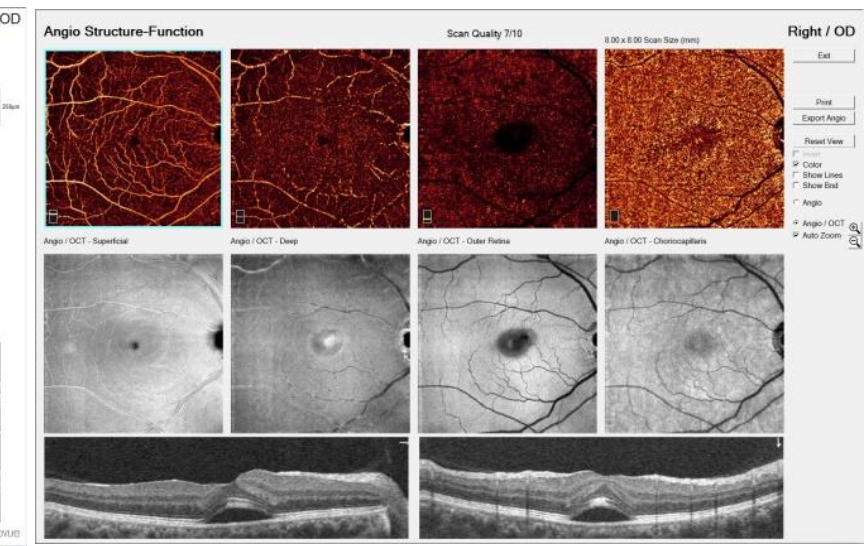

retinal detachment with the increased subfoveal choroidal thickness (403 micrometer) 
Omer Othman Abdullah; Saudi J Med Pharm Sci, June, 2020; 6(6): 449-451

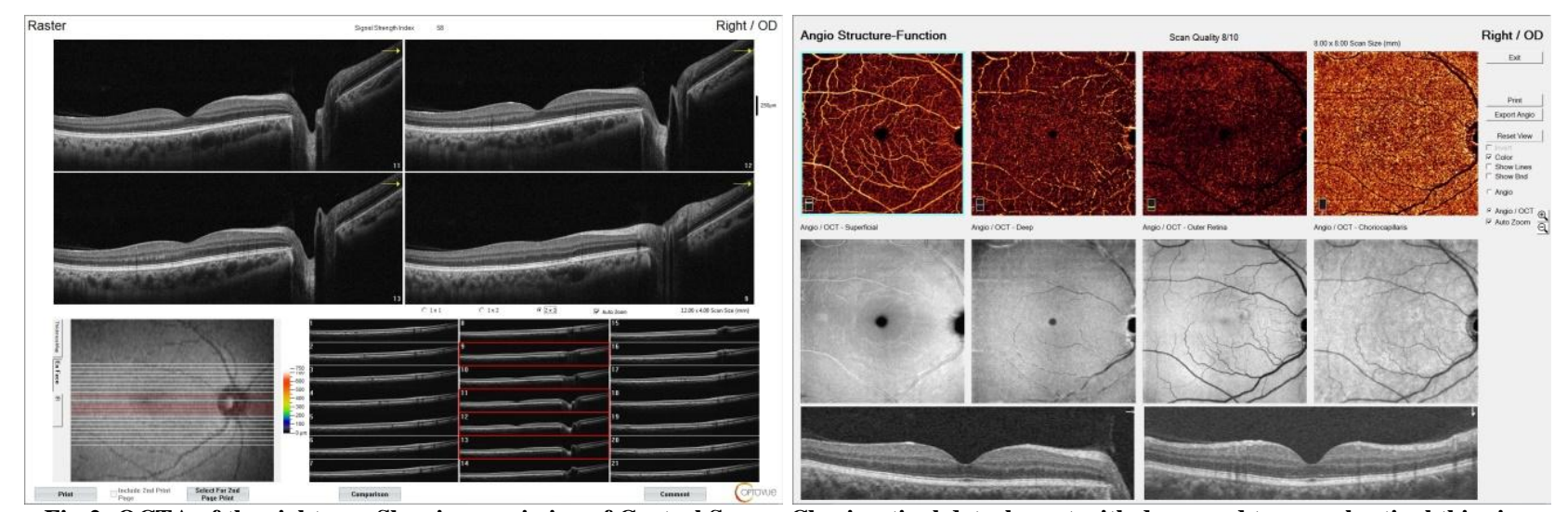

Fig-2: OCTA of the right eye. Showing remission of Central Serous Chorioretinal detachment with decreased temporal retinal thinning

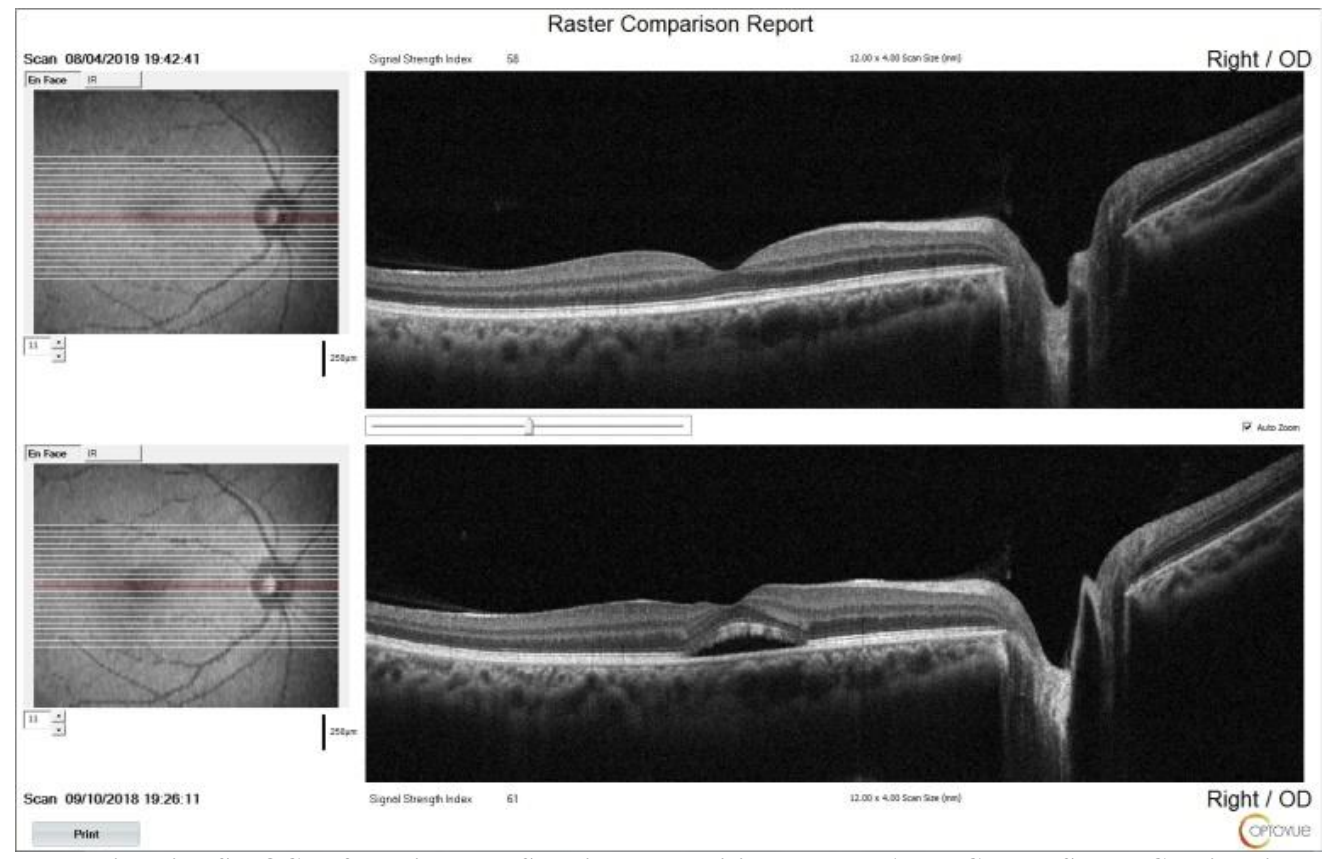

Fig-3: Enhanced-depth imaging SD-OCT of the right eye. Showing comparision between Acute Central Serous Chorioretinal detachment and remission in six months

\section{DiscuSSION}

In 1866, von Graefe had described this illness as central retinitis, while in 1922, Horniker postulated that the central retinitis due to capillary spasm, after that Gass named as central serous chorioretinopathy [1]. The acute central serous choroidopathy is whether associated with serous neurosensory retinal detachment or with a serous retinal pigment epithelial detachment in the posterior pole involving the macular region The illness known as Central Serous Detachment (CSR) when there is a neurosensory retinal detachment and called Central chorioretinopathy (CSC) when there is retinal pigment epithelium detachment which is caused by the serous fluid collection in the macular region, that affects healthy middle-aged, typically with spontaneous remission and had a unilateral tendency $[2,3]$ and affecting males more than females [4]. The actual cause remains unknown but persons with type A personality, having mental stress, using of steroids for any reason, Cushing's syndrome or tumors producing steroid and pregnancy regarded as risk factors [3]. The obstructive sleep apnoea Hereditary may play rule also [5].

In 1980, a case report was done for central serous retinopathy secondary to focal choroiditis in a 7 year-old girl by Fine and Owens and in 2012, a case report done by Kim et al. [3]. But our case is primary one based on the fundus, OCTA, the reports from pediatrician and endocrinologist; there were no any signs for secondary cause neither systemic nor local causes (in the retina and choroid).

The prognosis of visual recovery usually directly related to the visual acuity in the early stage of the illness and in the acute phase, the VA recovers within 2-3 months, Wong et al., [6].

The etiology of the central serous chorioretinopathy remains controversial. However, recently the hyperpermeability in the choroidal layer has been implicated which happens through cytokines; 
one of the most crucial cytokines is vascular endothelial growth factor (VEGF) that stimulates the hyperpermeability in the vascular layers, and more studies require to prove that. Another theory is stress, can have its effect on both catecholamines and cortisol levels; it causes an elevation in the serum level of epinephrine and norepinephrine; these changes observed in CSC patients which normalizes after recovery from the illness. Also, CSC patients, have an elevated serum and urinary cortisol, indicating stress involved in the etiology [4].

The patient did not get a recurrent attack and needed close checkup because the recurrence rate within one year is $50 \%$ [4].

His visual acuity recovered to $6 / 6$. The inner and outer retinal layers regained normal thickness. The reverse is postulated that a decrease in the outer retina can occur; also, a decreased photoreceptor layer was described [4].

\section{Conclusion}

The spontaneous remission and the absence of the secondary causes in favors the diagnosis of acute central serous chorioretinopathy.

\section{DeClarations}

Funding: None

Conflict of interest: None declared

\section{REFERENCE}

1. Nicholson, B., Noble, J., Forooghian, F., \& Meyerle, C. (2013). Central serous chorioretinopathy: update on pathophysiology and treatment. Survey of ophthalmology, 58(2), 103126.

2. Daruich, A., Matet, A., Dirani, A., Bousquet, E., Zhao, M., Farman, N., ... \& Behar-Cohen, F. (2015). Central serous chorioretinopathy: recent findings and new physiopathology hypothesis. Progress in retinal and eye research, 48, 82-118.

3. Kim, Y. C., Kim, S. D., \& Kim, K. S. (2012). A case of idiopathic central serous chorioretinopathy in a 12-year-old male treated with bevacizumab. Korean Journal of Ophthalmology, 26(5), 391-393.

4. Wang, M., Sander, B., La Cour, M., \& Larsen, M. (2005). Clinical characteristics of subretinal deposits in central serous chorioretinopathy. Acta ophthalmologica Scandinavica, 83(6), 691-696.

5. Weenink, A. C., Borsje, R. A., \& Oosterhuis, J. A. (2001). Familial chronic central serous chorioretinopathy. Ophthalmologica, 215(3), 183 187.

6. Wong, K. H., Lau, K. P., Chhablani, J., Tao, Y., Li, Q., \& Wong, I. Y. (2016). Central serous chorioretinopathy: what we have learnt so far. Acta ophthalmologica, 94(4), 321-325. 